\title{
Sutureless Phacovitrectomy in Vitreoretinal Diseases of Presbyopic Patients with Clear Crystalline Lens
}

\author{
Ahmed Hassan Abdel Wahab, Mostafa Mahmoud Mostafa, Ahmed Salah Abdel-Rehim \\ Department of Ophthalmology, Faculty of Medicin, Al-Azhar University \\ * Corresponding author: Ahmed Hassan Abdel Wahab, Mobile: (+20) 01001110348, E-mail: dr.hasan.wahab@live.com
}

\begin{abstract}
Background: Patients with vitroreinal diseases require surgical intervention frequently have coexisting lens opacification. Cataracts increase in prevalence with age, as do vitreoretinal pathologies such as macular holes, epiretinal membranes, and retinal detachments. Diabetes is another contributing factor that results in the development of both retinal disease and cataracts. In addition, vitrectomy itself causes cataracts to progress.

Objective: The aim of the work to review the results and complications of combined phacoemulsification and vitrectomy in presbyopic patients with clear crystalline lens and compare it with the results and complications of separate surgeries.

Patients and methods: This study carried on 40 presbyopic patients with clear lens having complex vitreoretinal disease in which vitrectomy is essentially required such as primary rhegmatogenous retinal detachement, recurrent rhegmatogenous retinal detachement, full thickness macular hole Stages 3,4,5, idiopathic epiretinal membrane, proliferative diabetic retinopathy and vitreous hemorrhage.

Result: There is no difference between the groups regarding sex, diagnosis, used material, VA, and IOP. VA showed improvement in both groups at the same degree post operatively. But there is no change in the IOP postoperatively compared to the preoperative measurement in both groups. The only complication that showed significant difference between the two groups is the visually significant PCO which occurred in 2 cases $(9.5 \%)$ in the Combined Phaco Vitrectomy compared to 10 cases of PSC in the PPV alone group (43.5\%).

Conclusion: Our study provides evidence that combing phaco and vitrectomy has more efficacy vitrectomy alone then phaco regarding visual acuity improvement with lower incidence of complications.

Keywords: Sutureless Phacovitrectomy, Vitreoretinal Diseases, Presbyopic Patients, Clear Crystalline Lens.
\end{abstract}

\section{INTRODUCTION}

Phacovitrectomy is now considered when lens opacities are mild or even nonexistent particularly in presbyopic patients. This could be called "nonessential" phacovitrectomy ${ }^{(1)}$.

Presbyopic eyes have lost the ability to accommodate, and this, combined with the inevitable postvitrectomy cataract, makes lens removal at the time of retinal detachment surgery desirable ${ }^{(2)}$.

Cataract surgery on vitrectomized eyes can be technically challenging due to the lack of vitreous support with a deep anterior chamber and "infusion deviation syndrome" with variable anterior chamber depth and pupil size ${ }^{(2)}$.

There are many advantages of the combined procedure such as; excellent visualization of peripheral retina, faster visual rehabilitation, avoiding the need for later cataract surgery, achieving emmetropia in many patients and reducing the cost as well as avoiding the difficulties of postvitrectomy cataract surgery ${ }^{(2)}$.

Phacovitrectomy also allows more complete anterior vitrectomy and access to the anterior retina and vitreous base without risking lens trauma. This allows a better gas fill, potentially improving tamponade of peripheral retinal breaks, especially inferiorly ${ }^{(3)}$.

Combining phacoemulsification and vitrectomy improves postoperative retinal visualization without the obscuring effect of the cataract, thus allowing accurate retinal assessment. Visual recovery is also not delayed by subsequent cataract development ${ }^{(4)}$.

However possible disadvantages of combined phacovitrevtomy include longer surgical time, increased risk of postoperative inflammation, slight increase in proliferative vitreoretinopathy rate, posterior capsule opacification and risk of iris/intraocular lens capture ${ }^{(2)}$.

\section{AIM OF THE WORK}

The aim of the work to review the results and complications of combined phacoemulsification and vitrectomy in presbyopic patients with clear crystalline lens and compare it with the results and complications of separate surgeries.

\section{PATIENTS AND METHODS}

This study carried in Al-Azhar University, AlHussein and Bab Al-Sharia Hospitals from January 2014 to March 2019 on 40 presbyopic patients, with clear lens having complex vitreoretinal disease in which vitrectomy is essentially required such as primary rhegmatogenous retinal detachement, recurrent rhegmatogenous retinal detachement, full thickness macular hole Stages 3,4,5, idiopathic epiretinal membrane, proliferative diabetic retinopathy and vitreous hemorrhage. 
Ethical approval and written informed consent: An approval of the study was obtained from Al- Azhar University academic and ethical committee. Every patient signed an informed written consent for acceptance of the operation.

\section{Exclusion criteria:}

- Non presbyopes.

- Cataractous patients.

- High refractive errors.

\section{Initial examination included:}

- Best corrected visual acuity.

- Slit lamp biomicroscopy examination of the anterior segment.

- Applanation tonometry to measure IOP.

- Dilated fundus examination.

Methods:

- Patients were divided into two groups, each of 20

The first group underwent combined phaco and vitrectomy.

- The patients underwent phaco procedures first using clear corneal wound.

- Intraocular lens implantation was delayed until the completion of posterior segment surgery.

- The IOL power was chosen directly from biometry of the affected eye when such measurements were possible. In cases when biometry in the affected eye wasn't not possible (macular off retinal detachment and dense vitreous haemorrhage), decisions on IOL power were based on biometry of the fellow eye.

- Pars plana vitrectomy was performed using a sutureless 20 -gauge or 23 -gauge vitrectomy system

The second group was vitrectomized first and followed up, once they develop cataract phaco surgery was done.

\section{Patients follow up:}

The patients were examined postoperatively at 1 day, 1 week, 1 month, 3 months, 6 months and 1 year for :

Posterior segment assessment and detecting complications such as recurrent detachment and proliferative vitreoretinopathy.

Posterior capsule opacification.

Fibrinous uveitis.

Refractive outcome.

\section{Statistical Analysis}

Descriptive statistics were used to describe the characteristics of the study participants and measurements during the study. Mean, standard deviations (SD), are reported for continuous variables. Frequencies with proportions are reported for categorical variables. Comparison of the numerical variables between the two groups is done using student $t$ test, while comparison of categorical variables is done using Chi square test and Fisher's exact test. Pre and post-operative measurements are compared using paired t test. Statistical significance was set at $\mathrm{p}<0.05$. Analysis was performed using IBM SPSS statistics, version 23 (IBM, Armonk, NY, USA).

\section{RESULTS}

\section{Demographic Characteristics}

Age of participants of the two groups is compared using student's $t$ test and there is significant difference in the age of the patients of the two groups. Age of the patient in the Combined Phaco Vitrectomy is higher than the PPV alone group. Mean age for the Combined Phaco Vitrectomy group is $57 \pm 6.2$ years while that for the other group is $48.2 \pm 7.6$ years. Table 1.

In the Combined Phaco Vitrectomy group there is one female and in the PPV alone group there is four females. There is no significant difference in the sex distribution between groups. Table 2 .

Table (1): Comparison of age of patients of the 2 groups

\begin{tabular}{|c|c|l|l|c|c|}
\hline \multicolumn{2}{|c|}{} & N & Mean & $\begin{array}{c}\text { Std. } \\
\text { peviation }\end{array}$ & P-value \\
\hline \multirow{2}{*}{ Age } & $\begin{array}{c}\text { Combined } \\
\text { Phaco } \\
\text { Vitrectomy }\end{array}$ & 21 & 57 & 6.2 & $<0.001$ \\
\cline { 2 - 5 } & PPV alone & 23 & 48.2 & 7.6 & \\
\hline
\end{tabular}

Table (2): patients' sex distribution in the groups.

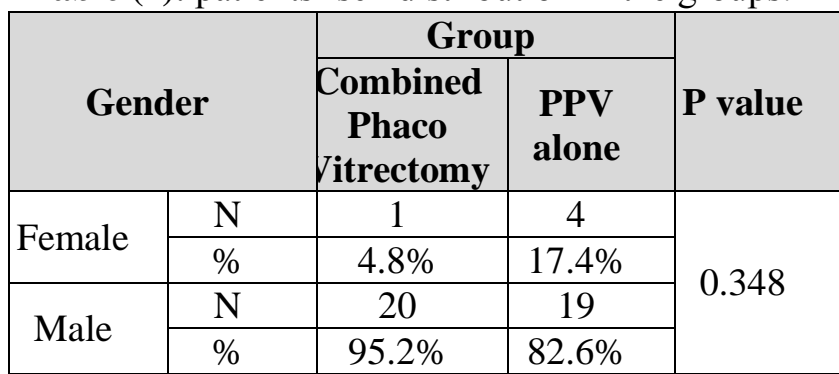

Surgery outcomes:

The visual acuity is ranked from best 1 representing: $20 / 20$ to 20 representing hand movement and the difference in VA rank before and 6 months after the operation. There is no significant difference between VA in the two groups before or after the operation and there is no difference in the improvement in VA in both groups. Also, there is no significant difference between IOP in the two groups before or after the operation and there is no significant difference in IOP before and after the operation in both groups. Table 3.

VA rank pre and post-operative is compared in both groups. Patients in both groups showed significant difference in VA before and after the operation. The mean difference in VA rank is 7 in the Combined Phaco Vitrectomy group while it is 5.7 in the PPV alone group representing the improvement in VA after the operation. IOP is compared pre and post- operative in both groups but there is no significant difference in IOP before and after the operation in any of the groups. Table 4. 
Table (3): comparison of VA and IOP in the two groups before, and the difference between before and after

\begin{tabular}{|c|c|c|c|c|}
\hline $\begin{array}{c}\text { Measurements of VA and } \\
\text { IOP }\end{array}$ & group & Mean & $\begin{array}{c}\text { Std. } \\
\text { Deviation }\end{array}$ & P-value \\
\hline \multirow{2}{*}{ PRE VA Rank } & Combined Phaco Vitrectomy & 12.0 & 5.8 & \multirow{2}{*}{0.380} \\
\hline & PPV alone & 10.4 & 6.2 & \\
\hline \multirow{2}{*}{ BCVA 6 m Rank } & Combined Phaco Vitrectomy & 5.0 & 3.6 & \multirow{2}{*}{0.843} \\
\hline & PPV alone & 4.8 & 3.7 & \\
\hline \multirow{2}{*}{ pre-op IOP } & Combined Phaco Vitrectomy & 13.9 & 1.6 & \multirow{2}{*}{0.884} \\
\hline & PPV alone & 13.8 & 1.9 & \\
\hline \multirow{2}{*}{ post-op IOP 6 months } & Combined Phaco Vitrectomy & 13.8 & 1.5 & \multirow{2}{*}{0.126} \\
\hline & PPV alone & 14.4 & 1.4 & \\
\hline \multirow{2}{*}{ VA Improvement } & Combined Phaco Vitrectomy & 7.0 & 5.3 & \multirow{2}{*}{0.363} \\
\hline & PPV alone & 5.7 & 4.8 & \\
\hline \multirow{2}{*}{ IOP difference ( post- pre) } & Combined Phaco Vitrectomy & -0.1 & 1.9 & \multirow{2}{*}{0.253} \\
\hline & PPV alone & 0.6 & 2.4 & \\
\hline
\end{tabular}

Table (4): Comparison of VA rank and IOP pre and post-operative in both groups

\begin{tabular}{|c|c|c|c|c|c|c|c|}
\hline & & & Mean & $\mathbf{N}$ & $\begin{array}{c}\text { Std. } \\
\text { Deviation }\end{array}$ & $\begin{array}{c}\text { Mean } \\
\text { Difference }\end{array}$ & $P$ value \\
\hline \multirow{4}{*}{ VA } & \multirow{2}{*}{$\begin{array}{l}\text { Combined } \\
\text { Phaco- } \\
\text { Vitrectomy }\end{array}$} & BCVA 6 m Rank & 5.0 & 21 & 3.6 & \multirow[b]{2}{*}{7.0} & \multirow[b]{2}{*}{$<0.001$} \\
\hline & & PRE VA Rank & 12.0 & 21 & 5.8 & & \\
\hline & \multirow{2}{*}{ PPV alone } & BCVA 6 m Rank & 4.8 & 23 & 3.7 & \multirow{2}{*}{5.7} & \multirow{2}{*}{$<0.001$} \\
\hline & & PRE VA Rank & 10.4 & 23 & 6.2 & & \\
\hline \multirow{4}{*}{ IOP } & \multirow{2}{*}{$\begin{array}{c}\text { Combined } \\
\text { Phaco- } \\
\text { Vitrectomy }\end{array}$} & $\begin{array}{l}\text { post-op IOP } 6 \\
\text { months }\end{array}$ & 13.8 & 21 & 1.5 & \multirow[t]{2}{*}{-0.1} & \multirow[t]{2}{*}{0.727} \\
\hline & & pre-op IOP & 13.9 & 21 & 1.6 & & \\
\hline & \multirow{2}{*}{ PPV alone } & $\begin{array}{l}\text { post-op IOP } 6 \\
\text { months }\end{array}$ & 14.4 & 23 & 1.4 & \multirow[t]{2}{*}{0.6} & \multirow[t]{2}{*}{0.235} \\
\hline & & pre-op IOP & 13.8 & 23 & 1.9 & & \\
\hline
\end{tabular}

\section{Surgery complications:}

There is no significant difference in the complication rate regarding post-op anterior chamber fibrinous reaction at 1 day $10 \%$ in the Combined Phaco Vitrectomy group vs. $0.0 \%$ in PPV alone group ( P value $=0.222$ ), post-op anterior chamber hyphaema at 1 day post-op $4.8 \%$ in the Combined Phaco Vitrectomy vs. $0.0 \%$ in PPV alone group ( $\mathrm{P}$ value $=0.477$ ), posterior synechia and pupillary membane at end first week post op $4.8 \%$ in the Combined Phaco Vitrectomy group vs. $0.0 \%$ in PPV alone group ( $\mathrm{P}$ value $=0.477$ ) and corneal oedema at 1st day post-op $10 \%$ in the Combined Phaco Vitrectomy group vs. $4.3 \%$ in the PPV alone group (P value=0.599). Significant difference observed in visually significant PCO 9.5\% in the Combined Phaco Vitrectomy group compared to 10 cases of PSC in the PPV alone group (43.5\%).Table 5.

Table (5): Comparison of complications occurrence in both groups

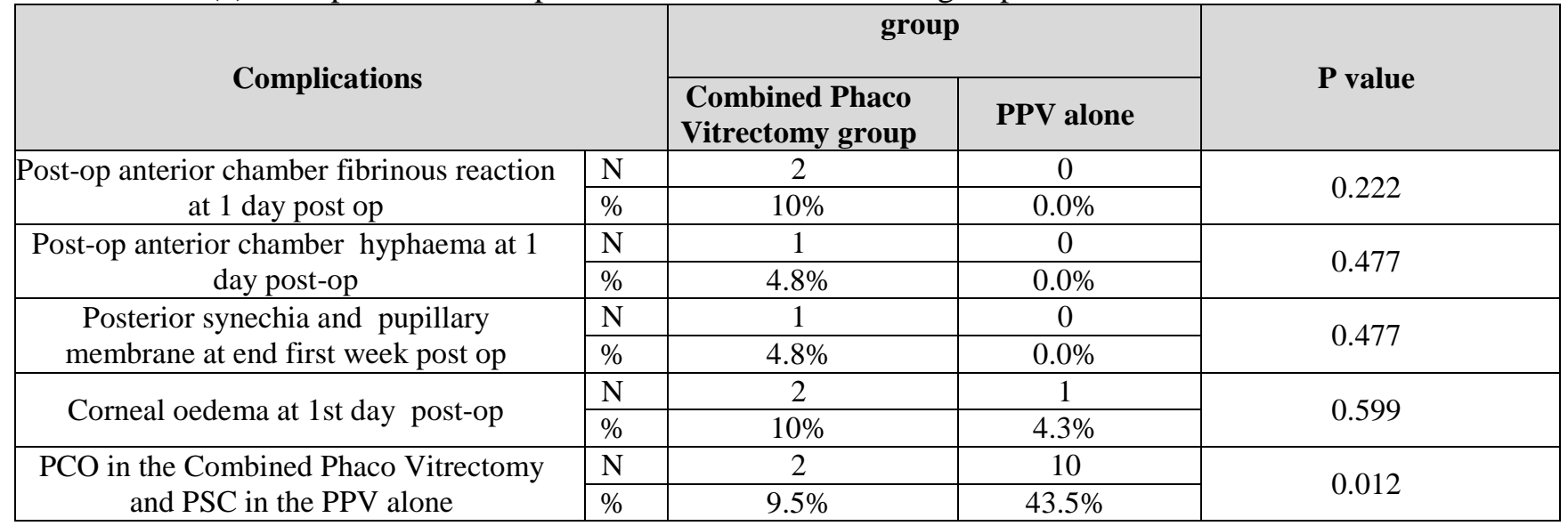




\section{DISCUSSION}

This study conducted a prospective analysis of 40 patients who combined phacoemulsification and vitrectomy or vitrectomy first then phacoemulsification. Combined group showed significant improvement on VA rank while IOP showed no significant difference. Regarding the complication combined group had visually significant PCO occurred in 2 cases $(9.5 \%)$ compared to 10 cases of PSC in the PPV alone group (43.5\%).

Tayyab et al. ${ }^{(5)}$ conducted prospective study with total of 40 eyes in which 20 eyes underwent combined phacoemulsification with $23 \mathrm{G}$ pars plana vitrectomy and another 20 eyes underwent pars plana vitrectomy alone in phakic patients. Study were conducted in tertiary care eye hospital in Lahore, Pakistan in the period between January 2016 to August 2016. Mean age of patients in both groups (53.1 \pm 9.61 years in combined group and 51.6 \pm 10.05 years in control group. In combined group, $11 / 20$ patients were male whereas in control 9/20 patients were male. Result showed the reasons for vitreoretinal intervention was rhegmatogenous retinal detachment followed by vitreous haemorrhage in combined study group.while our result showed Vitreous Hemorrhage was the commonest reson followed by Vitreous Hemorrhage then epiretinal membrane disease in the two groups.

Tayyab et al. ${ }^{(5)}$ showed combined group had significant difference in best corrected visual acuity preoperatively and post operatively. Regarding Intraocular pressure (IOP) was similar in both groups preoperatively with no significant difference .However, on 1st postoperative day, mean IOP in the combined was higher than in vitrectomy then phacoemulsification with p-value < 0.05 and at 6 months follow-up, there was no significant difference in mean IOP of both groups. While our result showed no significant difference in IOP neither preoperative nor post-operative.

Regarding occurrence of complications, Tayyab et al. (5) had $35 \%$ eyes in vitrectomy then phacoemulsification group developed visually significant cataract and $10 \%$ of eyes in combined group developed visually significant posterior capsular opacity with no incidents of posterior capsular rupture or IOL capture or IOL decentration . 1st day follow-up, combined group had $420 \%$ patients with AC fibrin reaction compared to only $5 \%$ patient in the other group with $\mathrm{p}<0.05 .10 \%$ in combined group had posterior synechie (PS) at end of 1st week follow-up and no PS in the other group (pvalue $<0.05$ ). Our result showed only significant difference in posterior capsule opacification, $9.5 \%$ in the combined group and $43.5 \%$ in the control group (pvalue $<0.05$ ). Post-operative anterior chamber fibrinous reaction at 1 day was $10 \%$ in the combined group and $0 \%$ in the control group while post-operative anterior chamber hyphaema at 1 day was $4.8 \%$ in the combined group and $0 \%$ in the control group but with no significant difference. Tayyab et al. ${ }^{(5)}$ explained the occurrence of anterior chamber inflammation was higher in the combined due to three out of four patients were diabetics who had undergone diabetic vitrectomy for vitreous hemorrhage and TRD and they used non branded ophthalmic viscoelastic device (OVD) and probable incomplete washout of OVD from $\mathrm{AC}$ at the end of surgery.

Canan et al. ${ }^{(6)}$ analyzed retrospectively medical records of 85 eyes of 85 patients with vitreous hemorrhage due to proliferative diabetic retinopathy (PDR) and coexisting cataract who underwent PPV in combination with phacoemulsification between January 2008 and September 2011. Forty patients were male and 45 were female. Their age ranged from 40 to 77 years with a mean of 59.6 years. The mean follow-up was 13 months, with a range of 6-48 months. The preoperative $\log$ MAR visual acuity changed from $2.62 \pm 0.6$ to $0.8 \pm$ 0.7 postoperatively. Postoperatively, visual acuity improved in 79 eyes (92.9\%), and did not change in six eyes $(7.1 \%)$. Intraoperative complications were transient corneal edema (five eyes) and posterior capsular rupture (one eye). Postoperative complications consisted of transient intraocular pressure elevation ( 25 eyes), corneal epithelial defects (six eyes), anterior chamber reaction (four eyes), hyphema (two eyes), posterior synechiae (four eyes), vitreous hemorrhage (23 eyes), retinal tears (five eyes), retinal detachment (one eye), and neovascular glaucoma (one eye). Canan et al. ${ }^{(6)}$ believe that the visual outcome and complications depended primarily on underlying posterior segment pathology and were not related to the combined procedure technique.

Jeoung et $\boldsymbol{a l} .{ }^{(7)}$ conducted prospective analysis on 154 patients who had phacoemulsification, IOL implantation, and PPV between September 2001 and August 2004 to evaluate factors affecting the refractive outcomes of combined procedures. Mean age of the patients was 60.9 years and the male-to-female ratio was 65:89. Result showed small biometric error after combined cataract and vitrectomy surgery, but it was within the tolerable range in most cases range in most cases. Cases with long axial lengths, poor preoperative visual acuity, and the preoperative foveal detachment developed myopic shifts.

Wensheng et al. ${ }^{(8)}$, conducted a retrospective analysis to assess the occurrence of complications in combining phacoemulsification and foldable intraocular lens (IOL) implantation with pars plana vitrectomy. It included 186 eyes of 149 patients with various vitreoretinal abnormalities and visually significant cataracts. Wensheng et al. ${ }^{(8)}$ showed $5.3 \%$ developed iatrogenic retinal hole , $3.2 \%$ developed transient corneal edema and $2.1 \%$ developed posterior capsule break. The main postoperative complication was posterior capsule opacification (21.5\%), elevated intraocularpressure $(9.7 \%)$, macular edema $(8.1 \%)$, fibrinous reaction $(6.9 \%)$, vitreous hemorrhage $(3.7 \%)$, posterior synechiae $(3.7 \%)$ and visual acuity improved by 3 lines or more on the Snellen chart in $87.1 \%$ of eyes.

Previous studies indicated that PCO was the most frequent complication of combined surgery. PCO is more common in children and in patients with diabetes or uveitis ${ }^{(9)}$. In combined anterior and posterior segment surgery, the occurrence of PCO may be greater due to the longer duration of surgery, increased manipulation, and greater inflammation that occurs in these eyes. In addition to the visual consequences, PCO may interfere with examination of the retinal periphery ${ }^{(10)}$. Wensheng $\boldsymbol{e t}$ al. 
(8) tried to decrease the occurrence of this complication by polishing the anterior capsule to remove residual epithelial cells and by using a hydrophobic acrylic IOL which has been reported to have a PCO rate as low as $2.2 \%$. The IOL material may play an important rolein PCO. Hollick et al. ${ }^{(11)}$ compared the effect of PMMA, silicone and acrylic IOL on PCO. The YAC capsulotomy rate was $0 \%$ for polyacrylic, $14 \%$ for silicone, and $20 \%$ for PMMA IOLs. Intraocular lenses made of polyacrylic were associated with a significantly lower YAG capsulotomy rate. The truncated edge of the acrylic IOL is believed to be responsible for the lower incidence of PCO. The barrier effect of the square optic edge, in addition to the material itself, may affect lens epithelial cell migration over the visual axis ${ }^{(11)}$. Wensheng et al. ${ }^{(8)}$ proposed if new IOLs with square-edge optics made from material other than acryl will also result in less PCO and lower YAG rates.

Wensheng et al. ${ }^{(8)}$ Recommended the use of constricting the pupil with a miotic at the conclusion of the procedure to avoid postoperative gas-related complications and suggest dilating gas-filled eyes once daily with short-actingagents, such as $1 \%$ tropicamide, rather than with long acting agents, such as atropine. As combining these agents with topical corticosteroids, may help prevent formation of synechiae and minimize the chance of pupillary capture by the IOL.

Our study has the following limitations. It was uncontrolled study with limited sample size. Most of previous studies were non comparative retrospective analysis with Few comparative, randomized studies were conducted on the topic. However, our design was prospective comparative study. Given that our results are similar to those of previous studies involving combined procedures. We propose that our results are reasonable. We recommend randomized clinical trial with larger sample size to be conducted to provide solid evidence on the effectiveness of combined procedures to overcome drawbacks mentioned studies taking into consideration operation materials used along with surgeon experience and underlying pathological conditions.

\section{CONCLUSION}

Our study is a prospective analysis that provides evidence of combing phaco and vitrectomy over vitrectomy alone then phaco. Our result in consistent with previous studies regarding that combing procedure provide higher improvement regarding visual acuity with no change in the IOP postoperatively compared to the preoperative measurement in both groups.

Our study insured equal patients' characteristics in both groups with no difference in groups regarding sex, diagnosis, used material, VA, and IOP. Age of the intervention group was statistically higher than the other group. The only complication that showed significant difference between the two groups is the visually significant PCO which occurred in 2 cases $(9.5 \%)$ in the Combined Phaco Vitrectomy group compared to 10 cases of PSC in the PPV alone group (43.5\%), while other complications showed no significant difference.

Previous studies show that anterior chamber inflammation and synechia development is a concern in combined procedures that require the use of aggressive steroids treatment despite our result showed that bi significant difference between the combined procedure groups and separate surgeries group regarding anterior chamber inflammation.

\section{REFERENCES}

1. Ling R, Simcock P, Mccoombes J et al. (2003): Presbyopic phacovitrectomy. The British Journal of Ophthalmology, 87: 1333-1335.

2. Smith M, Raman SV, Pappas G et al. (2007): Phacovitrectomy for primary retinal detachment repair in presbyopes. Retina, 27: 462-7.

3. Simcock $\mathbf{P}$ R, Scalia $\mathbf{S}$ (2001): Phacovitrectomy without prone posture for full thickness macular holes. $\mathrm{Br} \mathbf{J}$ Ophthalmol., 85: 1316-9.

4. Steel DH (2007): Phacovitrectomy: expanding indications. J Cataract Refract Surg., 33: 933-6.

5. Tayyab H, Khan AA, Javaid RM (2017): Clinical outcome of $23 \mathrm{~g}$ Trans-Conjunctival pars plana vitrectomy a prospective comparison of Phaco-Vitrectomy with only vitrectomy in phakic eyes. Pakistan Journal of Medical Sciences, 33: 1123-1127.

6. Canan H, Sizmaz S, Altan-Yaycioglu R (2013): Surgical results of combined pars plana vitrectomy and phacoemulsification for vitreous hemorrhage in PDR. Clin Ophthalmol., 7: 1597-601.

7. Jeoung JW, Chung H, Yu HG (2007): Factors influencing refractive outcomes after combined phacoemulsification and pars plana vitrectomy: results of a prospective study. J Cataract Refract Surg., 33: 108-14.

8. Wensheng $\mathrm{L}$, Wu R, Wang $\mathrm{X}$ et al. (2009): Clinical complications of combined phacoemulsification and vitrectomy for eyes with coexisting cataract and vitreoretinal diseases. Eur J Ophthalmol., 19: 37-45.

9. Wormstone IM, Wang L, Liu CS (2009): Posterior capsule opacification. Exp Eye Res., 88: 257-69.

10. Lam DS, Young AL, Rao SK et al. (2003): Combined phacoemulsification, pars plana vitrectomy, and foldable intraocular lens implantation. J Cataract Refract Surg., 29: 1064-9.

11. Hollick EJ, Spalton DJ, Ursell PG et al. (1999): The effect of polymethylmethacrylate, silicone, and polyacrylic intraocular lenses on posterior capsular opacification 3 years after cataract surgery. Ophthalmology, 106: 49-54. 